\title{
ON THE NITROGEN ABUNDANCE - AGE RELATION FOR MAIN SEQUENCE EARLY B-STARS
}

\author{
LEONID S. LYUBIMKOV \\ Crimean Astrophysical Observatory, Crimea, p.Nauchny, 334413, Ukraine
}

The results of the $\mathrm{He}, \mathrm{N}$ and $\mathrm{C}$ abundance determinations for MS early B-stars, summarized in the review [1], lead to the inference that there are evolutionary variations in atmospheric contents of these elements. The conclusion was made that CNO-cycle products emerge on a surface of B-stars during their MS phase yet and such appearance can be considered as a probable manifestation of some mixing process between outer layers and stellar interiors. In particular the $\mathrm{N}$ enrichment with the age was found, which reachs about 1.0 dex for most massive B-stars (see [1,2]). However Gies and Lambert [3] (further GL) investigated recently the $\mathrm{C}, \mathrm{N}$ and $\mathrm{O}$ abundances in a sample of early B-stars and did not confirm such great $N$ enrichment. Since the problem of early mixing is very important for the understanding of first evolutionary stages of massive stars, the GL data need further and thorough analysis.

It is necessary to take into account some essential circumstances. Firstly, the comparison of GL nitrogen abundances with earlier results [2] shows a systematic difference (Fig.1). The discrepancy is small enough for most of 16 common stars $(-0.17$ dex on the average, dashed line in Fig.1), but for spectroscopic binaries $\pi^{4} \mathrm{Ori}$ and $\pi^{5} \mathrm{Ori}$ it is about $-1.0 \mathrm{dex}$. It is possible that there is a marked influence of secondary components on spectra of these binaries. Moreover in the case of $\pi^{4} \mathrm{Ori}$ and $\pi^{5} \mathrm{Ori}$ there are great differences in used equivalent widths between GL and [2], and this can testify to probable spectral variability of both stars. Therefore these two stars must be excluded from further analysis.

Secondly, investigating the correlation between the nitrogen abundance log $\epsilon(N)$ and the stellar age $t$ we should take into account that errors in surface gravity $g$ have significant influence on $t$ determination. The comparison of GL $\log g$ values with results of Lyubimkov [2], Kane et al. [4] and Remie and Lamers [5] shows small systematic differences, which are correspondingly equal to 0.26 $\operatorname{dex}$ ( 14 common stars), $0.12 \mathrm{dex}$ (11 stars) and $0.21 \mathrm{dex}$ ( 7 stars) on the average. Consequently the values

$$
\begin{aligned}
& {[\Delta \log g]_{I}=\log g_{\mathrm{GL}}-\log g_{[2]}-0.26} \\
& {[\Delta \log g]_{I I}=\log g_{\mathrm{GL}}-\log g_{[4]}-0.12} \\
& {[\Delta \log g]_{I I I}=\log g_{\mathrm{GL}}-\log g_{[5]}-0.21}
\end{aligned}
$$


can be considered as residual discrepancies. These values are compared in Fig.2. We can see that discrepancies, which are greater than \pm 0.1 , evidently cannot be accidental and should be regarded as real errors in GL results. Corresponding corrections will be introduced into relation between the $\mathrm{N}$ abundance and the age.

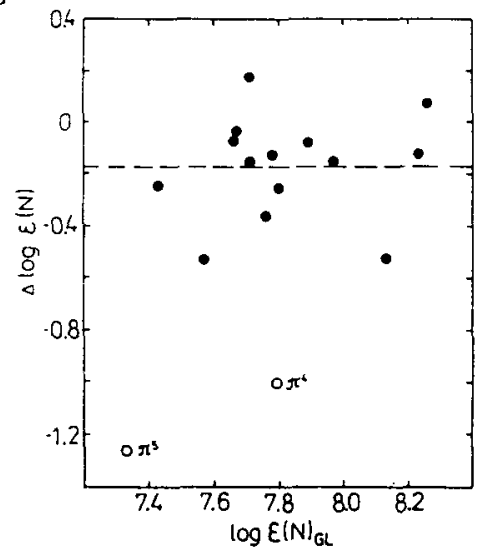

Fig.1. Difference in nitrogen abundances between GL data and our results [2]. Dashed line - the mean difference for all stars except $\pi^{4} O r i$ and $\pi^{5}$ Ori (open circles).

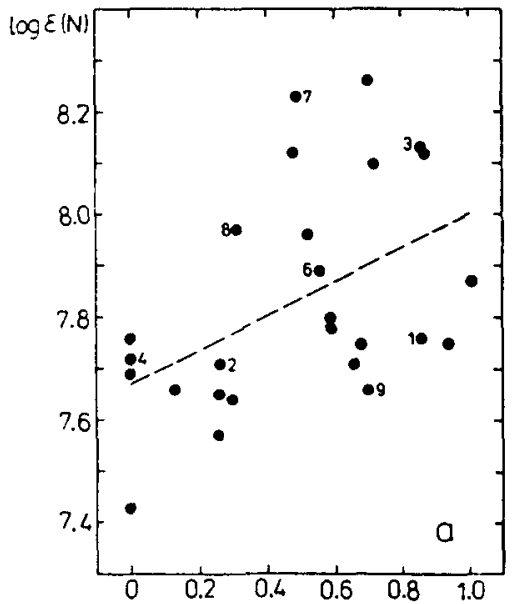

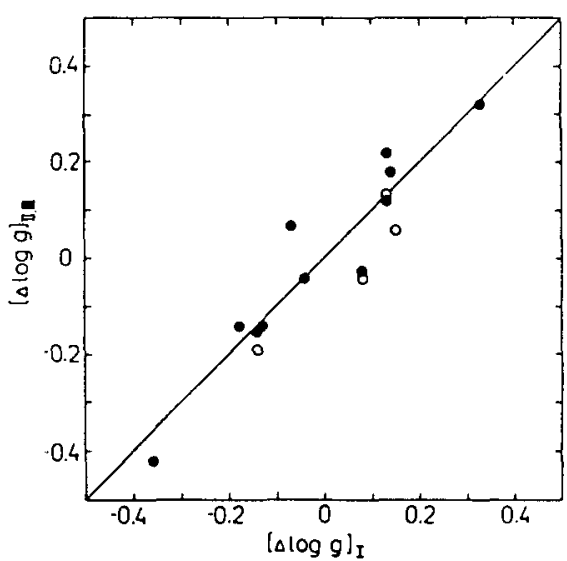

Fig.2.Residual discrepancies in $\log g$ calculated from formula (1) in com. parison with the va lues determined from (2) and (3) (filled and open circles correspondingly).

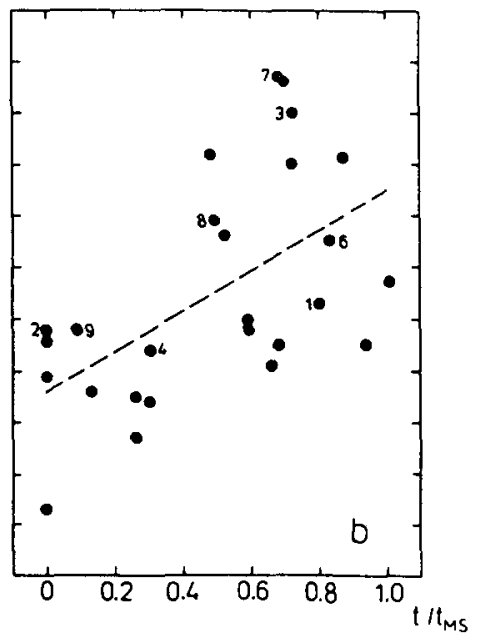

Fig.3. Nitrogen abundance in stars with masses $8.3-13.9 M_{\odot}$ as a function of age in the cases of incorrected (a) and corrected (b) $\log g_{G L}$ values for some stars. Dashed lines are drawn by the least squares method.

Thirdly, unlike previous works we will use the relative age $t / t_{\mathrm{MS}}$ instead of $t$ value, where $t_{\mathrm{MS}}$ is the lifetime on main sequence. Such substitution allows to 
reduce a scatter, which is due to a difference in masses (both the mass $M$ and the $t$ and $t_{\mathrm{MS}}$ values are determined from evolutionary tracks).

In Fig. $3 a$ we have plotted the $N$ abundance as a function of $t / t_{M S}$ for early B-stars with masses $M$ from $8.3 M_{\odot}$ to $13.9 M_{\odot}(26$ stars, the mean mass $\bar{M}=$ $\left.10.3 M_{\odot}\right)$. These stars are located in MS region as followed from evolutionary tracks [6] used in GL paper (all data in Fig.3a were taken from this paper, too). The dashed line drawn by the least squares method shows that $\log \epsilon(N)$ value increases by $0.33 \mathrm{dex}$, when $t / t_{\mathrm{MS}}$ changes from 0 to 1 .

For 8 stars the corrections to $\log g_{G L}$ are known (numbered dots in Fig.3). Corresponding corrections in $t / t_{\mathrm{MS}}$ and $\log \epsilon(N)$ values were found on a basis of calculations [6] and [7] (corrections in the $\mathrm{N}$ abundance are small). A new relation is shown in Fig. $3 \mathrm{~b}$. The nitrogen enrichment on the MS phase is 0.39 dex now. It is interesting how the correlation coefficient $q$ changes: it equals 0.36 for dependence on absolute age $t, 0.48$ for dependence on relative age $t / t_{M S}$ (Fig.3a), and 0.56 for relation with corrected $\log g$ values for some stars (Fig.3b). Consequently the correlation is strengthened.
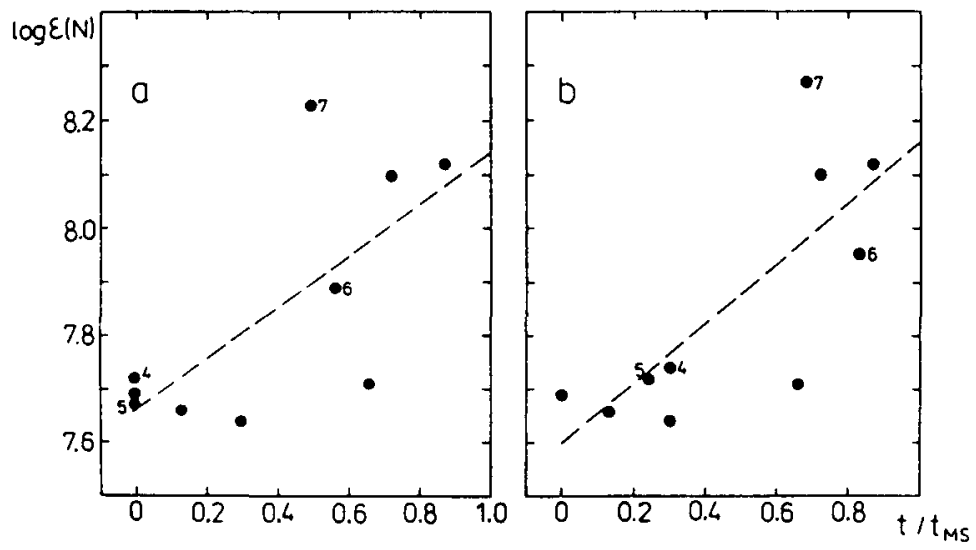

Fig.4. Same as Fig.3, but for stars with masses $11.1-14.8 \mathrm{M}_{\odot}$.

It is shown in [2] that the $\mathrm{N}$ enrichment intensifies with $M$ increase. In Fig.4 the more massive stars from the GL list are considered. Their masses $M$ vary from $11.1 M_{\odot}$ to $14.8 M_{\odot}\left(\bar{M}=12.5 M_{\odot}\right)$. This group consists of 10 stars, most of which were present already in Fig.3. In Fig.4 two cases are shown again: for incorrected (a) and corrected (b) $\log g_{\mathrm{GL}}$ values. The correction is included for 4 stars (numbered dots). The correlation coefficient is high enough in both cases: $q=0.70(\mathrm{a})$ and $q=0.76(\mathrm{~b})$. The nitrogen enrichment on the MS phase is 0.48 dex and 0.56 dex correspondingly; this is found from dashed lines in Fig.4 drawn by least squares method.

So we see that there is the nitrogen enrichment during the MS phase, which is about 0.4 dex for stars with mean mass $\bar{M}=10.3 M_{\odot}$ and almost 0.6 dex for 
stars with $\bar{M}=12.5 M_{\odot}$ (in more accurate case b). Both the correlation with age and the dependence on mass are confirmed. However a total $N$ enrichment is less than in our earlier paper [2]; for example, in the case of the star with $M=13 M_{\odot}$ the former value was about $1.0 \mathrm{dex}$ and the new value is $0.6 \mathrm{dex}$. The explanation is that in the $\mathrm{N}$ abundance determination by GL unlike [2] the differences in $\log g$ between investigated stars were taken into account. Another probable cause suggested by GL is different approach to the microturbulence. Meanwhile our analysis shows that this explanation is not suitable, because there is no correlation between microturbulent velocities (non-LTE values in GL paper) and age $t / t_{\mathrm{MS}}$.

Besides nitrogen abundance it is interesting to consider the $\mathrm{He}$ and $\mathrm{C}$ abundances obtained by GL. Our analysis of 24 B-stars with known $\mathrm{He} / \mathrm{H}$ values led to the conclusion that the $\mathrm{He} / \mathrm{H}$ value increases during the MS phase from 0.08 to 0.13 , i.e. by about 0.05 (for 7 stars the corrections in $\log g$ were taken into account). This result is in good accordance with previous one obtained for B-stars with masses $M=8-15 M_{\odot}$ (see $[1,8]$ ). Consequently GL data confirm our $\mathrm{He}$ abundance - age relation for MS early B-stars.

As concerns carbon abundance in these stars, in GL paper the mean value $\log \epsilon(C)=8.20$ was found, which is by 0.4 dex lower than solar abundance. In the review [1] general carbon underabundance of about 0.3 dex was noted. We can see a good agreement here, however according to GL data the observed $\mathrm{C}$ deficiency is unexpectedly independent of age $t / t_{M S}$. As pointed out in [1], there is a simple method for testing the $\log \epsilon(C)$ values: the total abundance $C$. $+\mathrm{N}$ must be approximately conserved during the MS phase and the following stage of yellow supergiants. For $26 \mathrm{~B}$-stars from the GL list the mean value is $\log \epsilon(C+N)=8.36 \pm 0.03$, whereas for supergiants from the list [9] it equals 8.62 (the latest solar value is 8.70 ). Obviously the condition is not fulfilled. Further investigations are needed to solve the problem of carbon underabundance in early B-stars.

\section{REFERENCES}

1. L.S.Lyubimkov. In Evolution of Stars: The Photospheric Abundance Connection, ed. G.Michaud and A.Tutukov, IAU Simp. N 145, Kluwer, Dordrecht, 1991, p.125.

2. L.S.Lyubimkov. Astrofizika, 20, 475, 1984.

3. D.R.Gies, D.L.Lambert. Ap.J. (in press).

4. L.Kane, C.D.McKeith, P.L.Dufton. Astron. Astrophys., 84, 115, 1980.

5. H.Remie, H.J.Lamers. Astron. Astrophys., 105, 85, 1982.

6. A.Maeder, G.Meynet. Astron. Astrophys. Suppl., 76, 411, 1988.

7. S.R.Becker, K.Butler. Astron. Astrophys. Suppl., 76, 331, 1988.

8. L.S.Lyubimkov. Astrofizilia, 29, 479, 1988.

9. R.E.Luck, D.L.Lambert. Ap.J., 298, 782, 1985. 